\title{
Longevity and Fecundity of Musca domestica (Diptera: Muscidae) as a Function of Temperature
}

\author{
M. G. FLETCHER, R. C. AXTELL, AND R. E. STINNER \\ Department of Entomology, North Carolina State University, \\ Raleigh, North Carolina 27695
}

\begin{abstract}
J. Med. Entomol. 27(5): 922-926 (1990)
ABSTRACT Longevity and fecundity of adult house flies were determined at constant temperatures of $20,25,30$, and $35^{\circ} \mathrm{C}$. At the four temperatures, respectively, the median mortality rates $(1 /$ days to $50 \%$ mortality) for females were $0.023,0.041,0.060$, and 0.099 and for males were $0.029,0.047,0.066$, and 0.085 . The numbers of days for $50 \%$ of the eggs to be deposited for each temperature were $34.5,21.3,10.2$ and 7.1 , respectively. The total numbers of eggs deposited per female for each temperature were 184, 729, 709, and 506, respectively. Models were developed for longevity and fecundity and their distributions as functions of temperature.
\end{abstract}

KEY WORDS Insecta, Musca domestica, longevity, fecundity

THE HOUSE FLY, Musca domestica L., is a major pest in animal production facilities, especially poultry houses (West 1951, Axtell 1985, Williams et al. 1985). Development of computer simulation models of house fly population dynamics is desirable to evaluate potential fly management strategies and tactics. The relationships of longevity and fecundity to temperature are critical to model development, but quantitative data on these relationships are surprisingly inadequate (West \& Peters 1973, Lysyk \& Axtell 1987, Weidhaas et al. 1977). The objective of our study was to describe the temperature-dependence of longevity and fecundity for use in a model of house fly population dynamics.

\section{Materials and Methods}

House flies were collected from a poultry house in North Carolina and had been in culture for about $1 \mathrm{yr}$ before initiating this study. Fly larvae were reared in a mixture of CSMA medium (RalstonPurina, St. Louis, Mo.), water, and yeast. Adults emerging over a 24 -h period were collected in three groups at 8 -h intervals. Fifty males and 50 females from each group were held in 4-liter mesh-top plastic containers at each of four constant temperatures $\left(20,25,30,35^{\circ} \mathrm{C}\right)$ in incubators $\left( \pm 1^{\circ} \mathrm{C}\right)$ with a 16:8 (L:D) photoperiod and about 30\% RH (maintained with a pan of water in each incubator). Thus, three replicates were established at each temperature over a 24-h period. Each container had a knitted cotton sleeve for access to retrieve dead flies and exchange food dishes. The food was diluted evaporated milk and granular sucrose in separate Petri dishes. Two cotton pads in the milk dish served as an oviposition substrate.

At 25,30 , and $35^{\circ} \mathrm{C}$, mortality and fecundity data were collected every $12 \mathrm{~h}$ for the first $11 \mathrm{~d}$ and every $24 \mathrm{~h}$ thereafter until all the flies in each container were dead. At $20^{\circ} \mathrm{C}$, adults were monitored every $24 \mathrm{~h}$ for the entire experiment. Mortality was monitored by removing and determining the sex of dead flies. Fecundity data were collected by removing the milk dish, washing all the eggs from the cotton pad onto a fine-mesh screen, and examining the pad under a dissecting microscope to assure that all eggs were removed. If $<500$ eggs were present, all eggs were counted directly. If $>500$ eggs were present, the number was estimated volumetrically using a calibrated $1-\mathrm{ml}$ pipette. The pipette was modified by opening the top of the squeeze bulb and heat-sealing the pipette end, creating a blind calibrated tube in which the height of the settled eggs could be measured. This procedure was calibrated eight times with different numbers of eggs ranging from 680 to 3,550 , with the result that $1 \mathrm{~cm}(0.12 \mathrm{ml})=1,062 \pm 56.4(\bar{x}$ $\pm \mathrm{SD})$ eggs, with a maximum error of $8.5 \%$.

\section{Data Analysis}

Mean longevity for each sex was calculated at each temperature by multiplying the number of flies that died each day by the number of days they had lived, summing these values, and dividing by the initial number of flies. The age at which mean oviposition occurred was calculated by multiplying the number of eggs laid each day by the day of oviposition, summing these values, and dividing by the total number of eggs laid. The mean number of eggs laid per female was calculated at each temperature by dividing the total number of eggs laid over the entire experiment by the initial number of females.

The cumulative distributions of female longevity 
and egg production at each temperature were calculated using the combined data from the three replicates. The cumulative proportion of eggs laid per living female $\left(c_{i}\right)$ up to day $i$ was calculated as:

$$
c_{i}=\frac{\sum_{t=1}^{t} E_{t}}{\sum_{t=1}^{m} E_{t}},
$$

where $m$ is maximum number of days of oviposition and $E_{t}$ is number of eggs per living female laid on day $t$.

For modeling purposes, median values rather than means were used. The median longevities (days) for males $\left(\mathrm{MT}_{50}\right)$ and females $\left(\mathrm{FT}_{50}\right)$, and the time (days) until the median number of eggs were deposited $\left(\mathrm{ET}_{50}\right)$, were determined. The corresponding rates for each of these were calculated as the inverse of the median days. These rates were related exponentially to temperature by:

$$
\mathrm{T}_{50} \text { rate }=\exp \left[\mathrm{b}_{1}\left(\mathrm{Temp}-\mathrm{b}_{2}\right)-1\right]
$$

where $b_{1}$ and $b_{2}$ were parameters estimated by the NLIN procedure (SAS Institute 1982).

Because both longevity and oviposition at a constant temperature are variable, both were modeled using the approach of Stinner et al. (1975). The distributions of longevity and oviposition were expressed as functions of the median physiological age. The scale of physiological age was set arbitrarily with $50 \%$ mortality or oviposition occurring at a physiological age of 1.0. It was assumed that the first individuals died or oviposited at some proportion (A) of median physiological age and $100 \%$ at another proportion $(B)$ of median physiological age. Equation 3 describes the cumulative proportion of the population dying (and the cumulative proportion of total eggs laid), $y$, at median physiological age $p$.

$$
y=(1-Z)^{k Z^{2}},
$$

where $Z=(B-\mathrm{p}) /(B-A)$. The parameter $k$ was estimated using the NLIN procedure (SAS Institute 1982). The goodness of fit was determined by linear regression of observed with predicted using REG procedure (SAS Institute 1982).

At each temperature, the mean fecundity (mean total eggs laid per female) was determined by summing the number of eggs laid per living female per day for all days. These data were fit to the poikilotherm model of Sharpe \& DeMichele (1977), with high temperature inhibition, which has the form:

$$
\begin{aligned}
& r(K)= \\
& \frac{\text { RH025 } \frac{K}{298.15} * \exp \left[\frac{\mathrm{HA}}{1.987}\left(\frac{1}{298.15}-\frac{1}{K}\right)\right]}{1+\exp \left[\frac{\mathrm{HH}}{1.987}\left(\frac{1}{\mathrm{TH}}-\frac{1}{K}\right)\right]}
\end{aligned}
$$

\begin{tabular}{|c|c|c|c|c|}
\hline \multirow{2}{*}{$\begin{array}{l}\text { Temp, } \\
{ }^{\circ} \mathrm{C}\end{array}$} & \multicolumn{2}{|c|}{ Longevity, d } & \multirow{2}{*}{$\begin{array}{l}\text { Age at mean } \\
\text { oviposition, } d\end{array}$} & \multirow{2}{*}{$\begin{array}{c}\text { Mean no. } \\
\text { eggs/óa }\end{array}$} \\
\hline & o & 8 & & \\
\hline 20 & $33.8 \pm 1.2$ & $44.6 \pm 4.2$ & $32.1 \pm 1.5$ & $117.8 \pm 36.5$ \\
\hline 25 & $21.9 \pm 1.0$ & $27.6 \pm 3.1$ & $18.1 \pm 2.0$ & $433.1 \pm 42.6$ \\
\hline 30 & $16.2 \pm 1.5$ & $17.5 \pm 0.4$ & $9.9 \pm 2.0$ & $494.9 \pm 73.2$ \\
\hline 35 & $12.4 \pm 1.3$ & $11.1 \pm 1.3$ & $6.7 \pm 0.7$ & $365.5 \pm 28.4$ \\
\hline
\end{tabular}

Table 1. Mean ( \pm SD) longevity, oviposition age, and number of eggs laid per female house fly per lifetime of three cohorts at four constant temperatures

${ }^{a}$ Total number of eggs divided by the initial female cohort size.

where $r(K)$ is the mean total number of eggs laid per female during her lifetime (fecundity) at temperature $K$ ( ${ }^{\circ}$ Kelvin $\left.={ }^{\circ} \mathrm{C}+273.15\right)$. $\mathrm{RH} 025$, HA, $\mathrm{TH}$, and $\mathrm{HH}$ are constants $(3,701,98,616,296$, and 110,181 , respectively) which we estimated by the nonlinear regression routine outlined by Wagner et al. (1984). The goodness of fit was determined by linear regression of the observed with the predicted values using the REG procedure (SAS Institute 1982).

\section{Results and Discussion}

Mean longevity, age at mean oviposition, and the mean number of eggs laid per female are presented in Table 1 . The mean longevities of males and females decreased with increasing temperature, with male longevities less than those of females. Female mean longevity ranged from 44.6 $\mathrm{d}$ at $20^{\circ} \mathrm{C}$ to $11.1 \mathrm{~d}$ at $35^{\circ} \mathrm{C}$, and mean eggs per female ranged from 117.8 at $20^{\circ} \mathrm{C}$ to 494.9 at $30^{\circ} \mathrm{C}$, with reduced oviposition at $35^{\circ} \mathrm{C}$. Age at mean oviposition decreased with increasing temperature. The only comparable data found in the literature was for $27^{\circ} \mathrm{C}$ (Lee et al. 1985). They reported a mean longevity for males of $17.6 \pm 2.6 \mathrm{~d}$ and 34.2 $\pm 4.3 \mathrm{~d}$ for females, with a mean number of eggs laid per female of $532.0 \pm 50.3$. Rockstein (1957) reported mean longevities at $27^{\circ} \mathrm{C}$ (two experiments) of 17.5 and $14.4 \mathrm{~d}$ for males and 32.7 and $30.8 \mathrm{~d}$ for females. The shorter mean female longevity which we found may have been caused by strain differences or diet or both.

The median rates for longevities of males and females $\left(\mathrm{MT}_{50}\right.$ and $\left.\mathrm{FT}_{50}\right)$, as well as the median rate for $50 \%$ egg deposition per female $\left(\mathrm{ET}_{50}\right)$, increased exponentially with increasing temperature (Table 2 ). The goodness of fit test of the three models had high $R^{2}$ values $(0.99,0.96$, and 0.99 , respectively), and the slopes and intercepts were not significantly different from 1 and 0 , respectively. The parameter estimates for Equations 2 and 3 are presented in Table 3.

To separate the effects of longevity and reproduction, mean fecundity (i.e., the total number of eggs laid per living female) was calculated as the cumulative sum of eggs laid per day divided by the sum of living females per day (Table 2). These fecundity values are, therefore, the total eggs per 
Table 2. Observed and predicted median rates $\left(\right.$ days $\left.^{-1}\right)$ for longevity of males ( $\left.\mathrm{MT}_{50}\right)$, females $\left(\mathrm{FT}_{50}\right)$, and egg deposition (ET $\mathbf{F o}_{50}$ ), and the mean fecundity for adult house flies held at four constant temperatures

\begin{tabular}{|c|c|c|c|c|}
\hline \multirow{2}{*}{ Parameters } & \multicolumn{4}{|c|}{ Temp, ${ }^{\circ} \mathrm{C}$} \\
\hline & 20 & 25 & 30 & 35 \\
\hline \multicolumn{5}{|l|}{$\mathrm{MT}_{50}$} \\
\hline Observed & 0.030 & 0.047 & 0.066 & 0.085 \\
\hline Predicted & 0.033 & 0.045 & 0.063 & 0.087 \\
\hline \multicolumn{5}{|l|}{$\mathrm{FT}_{50}$} \\
\hline Observed & 0.023 & 0.041 & 0.060 & 0.099 \\
\hline Predicted & 0.024 & 0.039 & 0.062 & 0.099 \\
\hline \multicolumn{5}{|l|}{$\mathrm{ET}_{50}$} \\
\hline Observed & 0.029 & 0.047 & 0.098 & 0.137 \\
\hline Predicted & 0.032 & 0.053 & 0.086 & 0.141 \\
\hline \multicolumn{5}{|l|}{ Fecundity ${ }^{a}$} \\
\hline Observed & 184 & 729 & 707 & 506 \\
\hline Predicted & 170 & 743 & 691 & 521 \\
\hline
\end{tabular}

${ }^{a}$ Mean fecundity is corrected for longevity (see text) and is the estimated maximum number of eggs a female could oviposit in its lifetime at that temperature.

female corrected for variable longevity. The maximum mean fecundity occurred at $25^{\circ} \mathrm{C}$, with predicted values close to the observed $\left(R^{2}=0.99\right.$; slope and intercept not significantly different from 1 and 0 , respectively).

The cumulative proportion of eggs laid per female per day for each temperature is illustrated in Fig. 1. When the chronological age is expressed as median physiological age (using Equation 2), the cumulative proportions of eggs laid per living female for each temperature (Fig. 2) can be described by a single equation (Equation 3). Likewise, the cumulative distributions of longevities had similar relationships to chronological and physiological age (Fig. 1 and 2). The goodness-of-fit test for the models of the cumulative distribution of male and female longevity and egg production had high $R^{2}$ values $(0.96,0.99$, and 0.96 , respectively). The slopes and intercepts were not significantly different from 1 and 0 , respectively.

These data were collected under laboratory conditions and represent maximum survival and fecundity of a wild strain of house flies that had been colonized for $1 \mathrm{yr}$. Colonization may have induced changes in survival and fecundity, as reported by

Table 3. Parameter estimates for rate equations

\begin{tabular}{crrc}
\hline \hline Parameters & o Longevity & \& Longevity & Egg deposition \\
\hline Equation 2 & & & \\
$b_{1}$ & 0.0645 & 0.0936 & 0.0982 \\
$b_{2}$ & 57.4088 & 49.0596 & 44.7618 \\
Equation 3 & & & \\
$A$ & 0.0831 & 0.1183 & 0.2840 \\
$B$ & 2.1449 & 2.4960 & 2.4527 \\
$K$ & 2.8904 & 1.8382 & 1.4967 \\
\hline
\end{tabular}
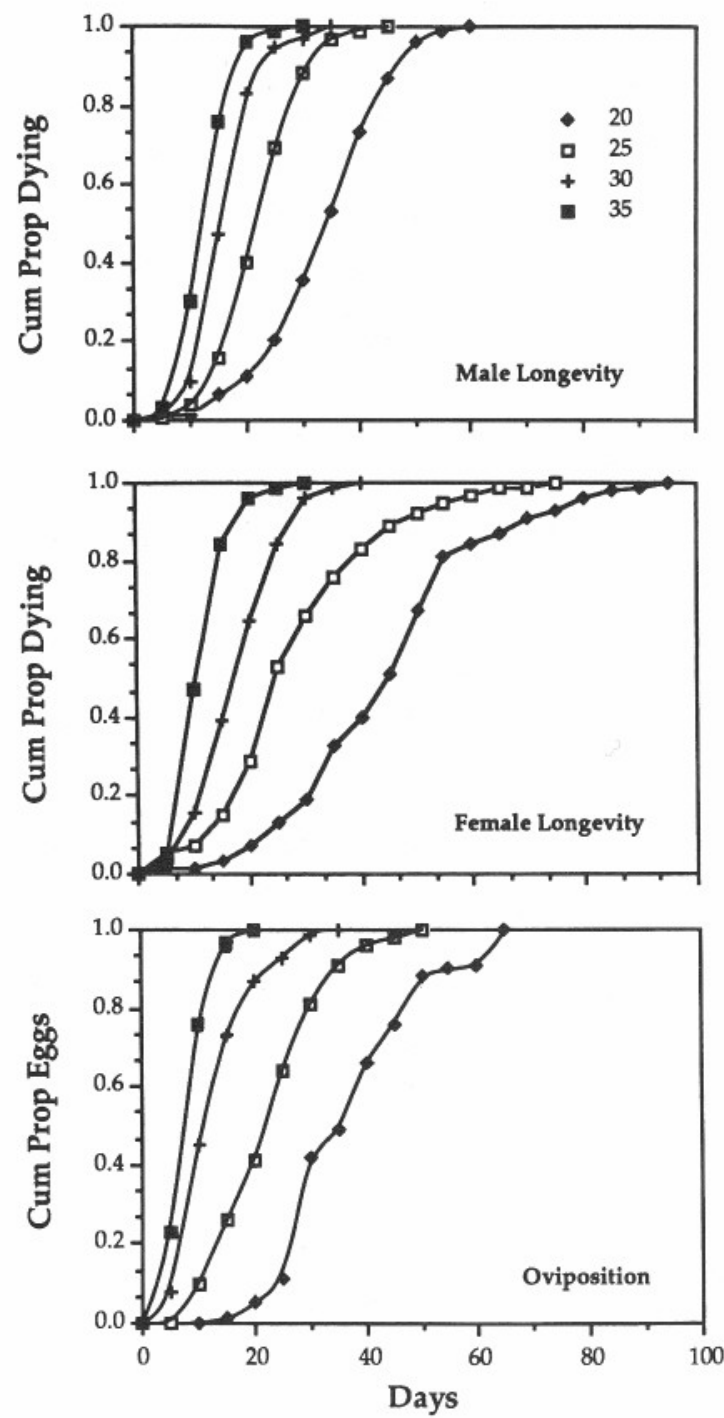

Fig. 1. Cumulative proportions of male and female house flies dying and cumulative proportion of eggs laid per female at four constant temperatures. $X$ axis is chronological age (d).

Elvin \& Krafsur (1984). The conditions under which the experiment was conducted may have caused positive or negative effects, but these are unknown. These effects were probably small in comparison with the large effects on survival and fecundity of conditions in the field; e.g., seasonal effects, larval and adult density, adult behavior, adult and larval nutrition (Ragland \& Sohal 1973, Black \& Krafsur 1987). Nevertheless, our data are valid estimates. As justified, our estimates may be altered on the basis of field data to reflect lower survival and fecundity in the wild.

Using the parameters we determined, these models of longevity and fecundity may be used to 

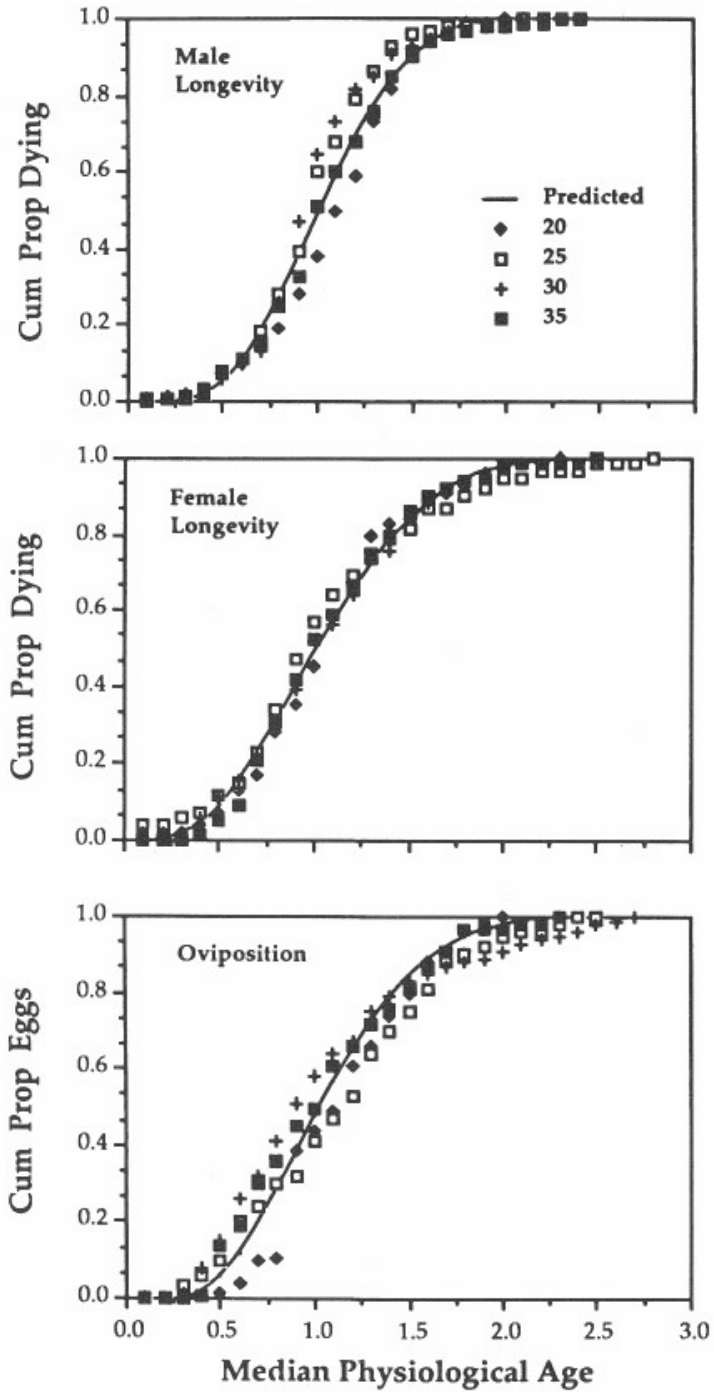

Fig. 2. Cumulative proportions of male and female house flies dying and cumulative proportion of eggs laid per female at four constant temperatures compared with simulated distributions based on median physiological age (1.0 physiological age equals 0.5 cumulative response).

calculate rates at any temperature and used in a population simulation model with variable temperature input reflecting field conditions. These models may be combined with other models, including those for the development of the immature stages of the house fly (Lysyk \& Axtell 1987) and for predator interactions (Geden \& Axtell 1988; Geden et al. 1988, in press), to construct computer simulation models of house fly population dynamics. Obviously, other factors affecting house fly populations must be included in a complete simulation model.

\section{Acknowledgment}

This is paper 12393 of the Journal Series of the North Carolina Agricultural Research Service, Raleigh. This research was supported in part by USDA-Cooperative States Research Service grants 86-CSRS-2-2889 and 89341-3-4240.

\section{References Cited}

Axtell, R. C. 1985 . Fly management in poultry production: cultural, biological, and chemical. Poultry Sci. 65: 657-667.

Black, W. C., IV \& E. S. Krafsur. 1987. Fecundity and size in the housefly: investigations of some environmental sources and genetic correlates of variation. Med. Vet. Entomol. 1: 369-382.

Elvin, M. K. \& E. Krafsur. 1984. Relationship between temperature and rate of ovarian development in the house fly, Musca domestica L. (Diptera: Muscidae). Ann. Entomol. Soc. Am. 77: 50-55.

Geden, C. J. \& R. C. Axtell. 1988. Predation by Carcinops pumilio (Coleoptera: Histeridae) and Macrocheles muscaedomesticae (Acarina: Macrochelidae) on the house fly (Diptera: Muscidae): functional response, effects of temperature, and availability of alternative prey. Environ. Entomol. 17: 739-744.

Geden, C. J., R. E. Stinner \& R. C. Axtell. 1988. Predation by predators of the house fly in poultry manure: effects of predator density, feeding history, interspecific interference, and field conditions. Environ. Entomol. 17: 320-329.

Geden, C. J., R. E. Stinner, D. A. Kramer \& R. C. Axtell. In press. MACMOD: a computer simulation model of Macrocheles muscaedomesticae (Acarina: Macrochelidae) population dynamics and rates of predation on immature house fly (Diptera: Muscidae). Environ. Entomol.

Lee, F. E., Jr., E. H. Bryant \& J. G. Baust. 1985. Fecundity and longevity of house flies after space flight. Experientia 41: 1191-1192.

Lysyk, T. J. \& R. C. Axtell. 1987. A simulation model of house fly (Diptera: Muscidae) development in poultry manure. Can. Entomol. 119: 427-437.

Ragland, S. S. \& R. S. Sohal. 1973. Mating behavior, physical activity and aging in the housefly, Musca domestica. Exp. Gerontol. 8: 135-145.

Rockstein, M. 1957. Longevity of male and female flies. J. Gerontol. 12: 253-256.

SAS Institute. 1982. SAS user's guide: statistics. SAS Institute, Cary, N.C.

Sharpe, P. J. H. \& D. W. DeMichele. 1977. Reaction kinetics of poikilotherm development. J. Theor. Biol. 64: 649-670.

Stinner, R. E., G. D. Butler, Jr., J. S. Bacheler \& C. Tuttle. 1975. Simulation of temperature-dependent development in population dynamic models. Can. Entomol. 107: 1167-1174.

Wagner, T. L., H. I. Wu, P. J. H. Sharpe, R. M. Schoolfield \& R. N. Coulson. 1984. Modeling insect development rates: a literature review and application of a biophysical model. Ann. Entomol. Soc. Am. 77: 208-225.

Weidhaas, D. E., D. G. Haile, P. B. Morgan \& G. C. LaBrecque. 1977. A model to simulate control of house flies with a pupal parasite, Spalangia endius. Environ. Entomol. 6: 489-500. 
West, L. W. 1951. The house fly. Comstock, Ithaca, N.Y.

West, L. S. \& O. B. Peters. 1973. An annotated bibliography of Musca domestica Linnaeus. Dawsons, London.
Williams, R. E., R. D. Hall, A. B. Broce \& P. J. Scholl. 1985. Livestock entomology. Wiley, New York.

Received for publication 23 October 1989; accepted 27 March 1990. 